\title{
Evolving Therapies for Fuchs Endothelial Dystrophy
}

Jodhbir Singh Mehta, 1,2,3 Yu Qiang Soh ${ }^{1,2,3}$ and Viridiana Kocaba ${ }^{1,4,5,6}$

1. Singapore Eye Research Institute (SERI), Singapore; 2. Singapore National Eye Center (SNEC), Singapore; 3. Eye-ACP, Duke-NUS Graduate Medical School, Singapore; 4. Department of Ophthalmology, Edouard Herriot Hospital, Hospices Civils de Lyon, Lyon, France;

5. Université de Lyon, Lyon, France; 6. Université Claude Bernard Lyon 1, Villeurbanne, France

\section{Keywords}

Fuchs endothelial dystrophy, FECD, regenerative therapy, cell therapy, tissue engineering, gene therapy

Disclosures: Jodhbir singh Mehta, Yu Qiang Soh and Viridiana Kocaba have nothing to disclosure in relation to this article.

Review Process: This article is an opinion piece and has not been submitted to external peer reviewers but was reviewed by the editorial board for accuracy before publication.

Authorship: All named authors meet the International Committee of Medical Journal Editors (ICMJE) criteria for authorship of this manuscript, take responsibility for the integrity of the work as a whole, and have given final approval to the version to be published.

open Access: This article is published under the Creative Commons Attribution Noncommercial

License, which permits any noncommercial use, distribution, adaptation, and reproduction provided the original author(s) and source are given appropriate credit. (c) The Authors 2018.

Received: 20 July 2018

Published Online: 13 August 2018

Citation: European Ophthalmic Review. 2018:12(1):26-8

Corresponding Author: Jodhbir S Mehta,

Singapore Eye Research Institute, 20 college

Road, The Academia Discovery Tower Level 6 ,

singapore 169856. E: jodmehta@gmail.com

Support: No funding was received for

the publication of this article
Fuchs endothelial dystrophy (FED) is a corneal endothelial disease characterised clinically by corneal endothelial dysfunction and guttae excrescences on the posterior corneal surface. The guttae excrescences represent foci of thickened posterior collagenous layers. The demise of healthy corneal endothelial cells (CECS) in FED leads to impairment of the endothelial pump mechanism, resulting in the progressive worsening of corneal oedema. In FED, corneal oedema and the presence of guttae results in compromised visual acuity, reduced contrast sensitivity and degradation of visual quality by the induction of higher order aberrations even in the early stages of the disease. Patients with mild FED are usually managed conservatively with the application of topical hypertonic saline eye drops. For patients with severe FED, keratoplasty has been the only therapeutic option. Replacement of diseased CECs by a healthy donor graft allows restoration of endothelial pump activity and improvement in corneal oedema.

While penetrating keratoplasty used to be the only surgical method available to treat end-stage FED, endothelial keratoplasty (EK) is now considered the standard of care. The most commonly practiced forms of EK are Descemet-stripping-automated EK and Descemet membrane EK. A global survey of 283,530 corneal transplants performed in 116 different countries in 2012 showed that FED was the leading indication for all corneal transplantations (accounting for 39\%). ${ }^{1}$

\section{Evolving therapies}

Primary descemetorhexis or Descemet stripping without endothelial keratoplasty

There have been several reports of CEC repopulation and corneal clearance originating from the peripheral descemetorhexis margins in patients who have undergone Descemet membrane EK for FED. The most plausible explanation was that the host CECs migrated from their peripheral locations toward the central area of bare stroma to restore anatomical integrity of the endothelium. ${ }^{2}$ In view of the fact that patients with FED retain a peripheral rim of healthy CECS, it was suggested that stripping of the central, diseased Descemet membrane without transplantation of an endothelial graft ('primary descemetorhexis' or 'Descemet stripping without EK') may be a possible therapeutic alternative for patients with FED.

In general, the outcomes of studies investigating the efficacy of primary descemetorhexis for the treatment of FED have been inconsistent, which may be partly attributed to heterogeneity in patient characteristics and the small number of subjects assessed in each study. We recently performed a systematic analysis of all published cases to understand some trends in outcomes. ${ }^{3}$ Three features may explain the variability in results: (i) age - there is a trend that advanced age (>60 years) is associated with poorer visual outcomes; (ii) the size of the distance the CECs must migrate - the larger the descemetorhexis the less chance of recovery; and (iii) genetics - FED is known to be associated with a spectrum of genetic abnormalities, most commonly an expanded CTG repeat sequence in TCF4. Variation in the trinucleotide repeat expansion length, either due 
to inter-individual differences or an age-dependent expansion bias, may be associated with a commensurate dysfunction of TCF4, which encodes for transcription E2-2, thus leading to differences in CEC migration rates.

The practicality of primary descemetorhexis as a treatment modality for FED is also premised upon long-term survival and functional potential of the redistributed corneal endothelium. The patients wouldn't require long-term steroids but longer-term cell attrition of the migrated CECs needs further investigation.

\section{Pharmacological adjuncts Rho kinase inhibitors}

As mentioned, primary descemetorhexis for the treatment of FED is more likely to succeed in younger patients. However, the majority of patients with FED who are symptomatic and require treatment, are elderly. In recent years, there has been mounting interest in the use of Rho kinase (ROCK) inhibitors for the treatment of corneal endothelial diseases. ROCK inhibitors have demonstrated efficacy in the stimulation of corneal endothelial regeneration via enhancement of $\mathrm{CEC}$ proliferation, migration and adhesion in in vitro, in vivo animal models and in ex vivo studies on human cadaver tissue. ${ }^{4,5}$ Clinically, ROCK inhibitors were shown to accelerate CEC migration and regeneration of the corneal endothelial monolayer in four patients with FED. ${ }^{6}$ The patients first underwent transcorneal cryotherapy to remove the central region of diseased endothelium followed by intensive application of a topical ROCK inhibitor. Three out of four patients experienced improvements in central corneal oedema. Even though these results are encouraging, this approach does not remove the diseased Descemet membrane or its associated guttae, which are also known to contribute toward higher-order aberrations and degradation in the quality of vision.

\section{Antioxidants}

CECs are constantly exposed to high levels of both endogenous and exogenous oxidative stress secondary to continuous environmental ultraviolet radiation exposure and heightened metabolic demands in maintaining the endothelial pump. Evidence has emerged to suggest the importance of oxidative stress in the pathogenesis of FED. ${ }^{7}$ Hence, pharmacological agents which protect against oxidative stress may possibly confer a survival advantage to CECs in patients with FED. Preliminary studies with in vitro animal CEC culture models have demonstrated the therapeutic potential of mefenamic acid and oxotremorine in this respect. ${ }^{8}$ Human CEC culture experiments have also indicated that L-ascorbic acid 2-phosphate promotes CEC population expansion via its antioxidant properties. ${ }^{9}$ To date, there are no clear clinical guidelines to support the routine use of these agents for the treatment of FED and further work is required to validate the efficacy of these agents in humans.

\section{Descemet membrane transplant}

The advantage of primary descemetorhexis is that it allows for removal of the diseased Descemet membrane and its associated guttae. However, Descemet stripping alone results in an environment that is not conducive for CEC migration and regeneration of the corneal endothelial monolayer, as shown by ex vivo human studies. ${ }^{5}$ Both ex vivo and animal studies have shown that the transplantation of an acellular Descemet membrane, following a primary descemetorhexis will provide a more conducive environment for CEC migration. ${ }^{5,10}$ This has been successfully demonstrated in clinical cases in patients below the age of 60 years. ${ }^{11}$ In patients over 60 years, a topical ROCK inhibitor will need to be applied to improve cell migration in addition to the Descemet membrane transplant surgery. This is currently part of an ongoing clinical study (ClinicalTrials.gov Identifier: NCT03275896). ${ }^{12}$

\section{Cell therapy}

The possibility of primary descemetorhexis augmented by pharmacological adjuncts and Descemet membrane transplant as a therapeutic modality for FED is premised on the assumption that a healthy rim of CECs exists in the periphery of the corneal endothelium. Therefore, such an approach may not be feasible in severe cases of FED. In such cases, cell therapy modalities, in which engineered CECS are introduced on the posterior corneal surface to replace or reform the CEC layer, may be more appropriate. Over the past decade, significant improvements have been made in the field of human CEC culture and have resulted in significant improvements to the quality and quantity of cultured human CECS. Treatment of FED by the delivery of these cells to the diseased host endothelium, either by direct intracameral injection or via a carrier, has demonstrated great potential as scientifically and economically viable alternatives to standard keratoplasty.

In a cell-carrier approach, synthetic or biological tissue engineered EK can be constructed by seeding of the cultured CECS at a physiological density onto a thin carrier. The tissue engineered EK can subsequently be transplanted onto a Descemet membrane-stripped posterior corneal surface in a manner similar to Descemet stripping EK. Experimental data from animal models have been encouraging and institutional review board approval has already been obtained to validate the safety and efficacy of this technique in a clinical trial at our centre. ${ }^{13}$

The alternative approach of intracameral cell injections was first reported by Okumura et al. in 2014. ${ }^{14}$ The endothelial layer of each monkey eye was denuded, while preserving the integrity of the Descemet membrane. This was followed by intracameral injection of a suspension of cultured monkey CECs supplemented with a ROCK inhibitor, combined with a strict face-down posturing regime for 3 hours. The role of intracameral cell injections in the treatment of FED is currently being assessed in a human clinical trial in Japan (Clinical Trial Registration Number: UMIN000012534). ${ }^{15}$ The outcomes of this study have recently been published showing successful restoration of the corneal endothelial monolayer in 11 patients. ${ }^{16}$ However, considering that Descemet membrane guttae in FED corneas may result in significant physical impediment to the adhesion of CECS, it is unclear if the strategy of intracameral cell injection without Descemet membrane stripping may result in the formation of a stable endothelial monolayer, especially in the presence of dense and large guttae. Further work is required in this context.

\section{Gene therapy}

Over the past two decades, significant advances have been made in our understanding of trinucleotide repeat expansion diseases. As it is now known that FED is, in a significant proportion of cases, a trinucleotide repeat disease, gene therapy modalities could theoretically be applicable for patients with FED as well.

A wide spectrum of modalities exist for gene therapy. Anomalous DNA segments may possibly be directly removed by endonucleases such as transcription activator-like effector nucleases (TALENS). Single-strand RNA oligonucleotides may be designed to inhibit mRNA transcription by 
binding the promoter sequence or to inhibit translation by interfering with the translation initiation complex. In particular, significant progress has been made in the development of single-stranded, antisense oligonucleotide (AON)-based therapeutics for modifying splicing patterns of pre-mRNA molecules by steric blockage. ${ }^{17}$ This produces less pathological mRNA transcripts and induces cleavage of the AON-mRNA duplex by endogenous RNase-H, in order to eliminate the trinucleotide repeat sequence. Moving forward, it may be possible to design viral-vector delivered CRISPR-Cas9 endonucleases for in vivo delivery via intracameral injections to achieve genomic deletion of the CTG trinucleotide repeat expansion sequence and obliteration of the deleterious FED phenotype.

\section{Conclusion}

Therapeutic modalities for FED have evolved significantly over the past few decades. The prevailing belief has been that the corneal endothelium does not possess regenerative capabilities and that corneal endothelial transplantation is the only definitive treatment for FED. However, an improved understanding of the biology of CECS and the genetics underlying FED have led to the design of multiple alternative therapeutic modalities, many of which are premised upon the stimulation of CEC regeneration and/or migration. While most of this work is currently experimental in nature, some approaches have successfully made it to the stages of clinical trial validation. This is a rapidly developing field and we expect significant changes over the next decade.
1. Gain P, Jullienne R, He Z, et al. Global survey of corneal transplantation and eyebanking. JAMA Ophthalmolol. 2016,134:167-73.

2. Dirisamer M, Dapena I, Ham L, et al. Patterns of corneal endothelialization and corneal clearance after descemet membrane endothelial keratoplasty for fuchs endothelial dystrophy. Am J Ophthalmol. 2011;152:543-55.

3. Soh YQ, Peh GS, Mehta JS. Evolving therapies for FECD. Regen Med. 2018;13:97-115

4. Okumura N, Koizumi N, Ueno M, et al. ROCK inhibitor converts corneal endothelial cells into phenotype capable of regenerating in vivo endothelial tissue. Am J Path. 2012:181:268-77

5. Soh YQ, Peh G, George BL, et al. Predicative factors for corneal endothelial cell migration. IOVS. 2016;57:338-48.

6. Okumura N, Koizumi N, Ueno M, et al. The new therapeutic concept of using rho kinase inhibitor for the treatment of corneal endothelial dysfunction. Cornea. 2011;30:S54-59.

7. Jurkunas UV, Bitar MS, Funaki T, Azizi B. Evidence of oxidative
Stress in the pathogenesis of FECD. Am J Path. 2010;177:2278-89. 8. Kim EC, Toyono T, Berlinicke CA, et al. Screening and characterization of drugs that protect corneal endothelial cells against unfolded protein response and oxidative stress. IOVS. 2017; $58: 892-900$

9. Shima N, Kimoto M, Yamaguchi M, Yamagami S. Increased proliferation and replicative lifespan of isolated human cornea endothelial cells with L-ascorbic acid 2-phosphate. IOVS. 2011;52:8711-7.

10. Bhogal $M, L$ win $C N$, Seah $X-Y$, et al. Allogeneic Descemet's membrane transplantation enhances corneal endothelial membrane transplantation enhances corneal endothelial
monolayer formation and restores functional integrity following monolayer formation and restores functiona

11. Soh YQ, Mehta JS. Regenerative therapy for FECD. Cornea 2018;37:523-7.

12. Clinicaltrials.gov. Evaluation of the Efficacy of Descemet Membrane Transplantation for the Treatment of Fuchs' Endothelial Dystrophy. ClinicalTrials.gov Identifier: NCT03275896. Available at: https://clinicaltrials.gov/ct2/show/NCT03275896 (accessed 30 July 2018).

13. Peh GS, Ang HP, Lwin CN, et al. Regulatory compliant tissueengineered human corneal endothelial grafts restore cornea function of rabbits with bullous keratopathy. Sci Report. 2017:12149

14. Okumura N, Kinoshita S, Koizumi N. Cell-based approach for treatment of corneal endothelial dysfunction. Cornea. 2014;33:S37-41

15. UMIN-CTR Clinical Trial. Clinical application of corneal endothelial regenerative medicine by means of cultured human corneal endothelial cell transplantation. UMIN000012534. Available at: https://upload.umin.ac.jp/cgi-open-bin/ctr_e/ Available at: https://upload.umin.ac.jp/cgi-open-bin/ctr_e/
ctr_view.cgi?recptno=R000014592 (accessed 30 July 2018)

Ctr_view.cgi? recptno=R000014592 (accessed 30 July 2018).
16. Kinoshita S, Koizumi N, Ueno M, et al. Injection of cultured cells Kinoshita S, Koizumi N, Ueno M, et al. Injection of cultured ce.
with a ROCK inhibitor for bullous keratopathy. N Engl I Med. 2018;378:995-1003

17. Gao Z, Cooper TA. Antisense oligonucleotids: rising stars in eliminating RNA toxicity in myotonic dystrophy. Hum Gene Ther. 2013;132:499-507 